\title{
Projection Selection Dependency in Binary Tomography*
}

\author{
László Varga ${ }^{\dagger \ddagger}$ Péter Balázs ${ }^{\dagger}$ and Antal Nagy ${ }^{\dagger}$
}

\begin{abstract}
It has already been shown that the choice of projection angles can significantly influence the quality of reconstructions in discrete tomography. In this contribution we summarize and extend the previous results by explaining and demonstrating the effects of projection selection dependency, in a set of experimental software tests. We perform reconstructions of software phantoms, by using different binary tomography reconstruction algorithms, from different equiangular and non-equiangular projections sets, under various conditions (i.e., when the objects to be reconstructed undergo slight topological changes, or the projection data is affected by noise) and compare the results with suitable approaches. Based on our observations, we reveal regularities in the resulting data and discuss possible consequences of such projection selection dependency in binary tomography.
\end{abstract}

Keywords: discrete tomography, reconstruction, adaptive projection acquisition, GPU-accelerated computing, non-destructive testing

\section{Introduction}

The main goal of transmission tomography is to reconstruct the inner structure of given objects from a set of their projections. This is usually done by exposing the object of study to some electromagnetic or particle radiation at one side and measuring the amount of received energy on the other end. After the projections have been gathered, one can apply certain reconstruction algorithms for discovering the linear attenuation coefficients of the object at its different points.

The reconstruction can be performed in many ways. In the ideal case (when we have hundreds of projections available) one can use, e.g., the filtered backprojection

\footnotetext{
*This research was in part supported by the TÁMOP-4.2.2/08/1/2008-0008 program of the Hungarian National Development Agency, by the TÁMOP-4.2.1/B-09/1/KONV-2010-0005 project co-financed by the European Union and the European Regional Development Fund, and by the János Bolyai Research Scholarship of the Hungarian Academy of Sciences.

${ }^{\dagger}$ Department of Image Processing and Computer Graphics, University of Szeged, Szeged, Hungary,E-mail: \{vargalg, pbalazs, nagya\}@inf .u-szeged.hu

$\ddagger$ Corresponding author.
} 
method or other continuous techniques for finding the reconstruction of arbitrary objects $[7,12]$. Unfortunately, acquiring hundreds of projections is sometimes impossible, since taking too many of them can be expensive or can even damage the object of study. In this case, one can try to improve the quality of the reconstruction basically in two ways. One approach is to take the projections having the highest information content in order to get sufficient information from fewer projections [15]. Another common approach is to develop more accurate reconstruction algorithms by using some prior information of the objects of interest.

In discrete tomography $[8,9]$ we assume that the object to be reconstructed consists of only a few (usually 2 to 4 ) materials, having known attenuation coefficients. With such a strong prior information, algorithms have been developed capable of producing accurate reconstructions from a limited amount of (say, up to 10) projections. However, the low number of projections gives a relatively high freedom in choosing the directions to take projections with.

Our previous studies $[14,17,18,19]$ revealed that different projection sets of an object can have entirely different information content, some holding more or less information than others. Despite their good performance, discrete reconstruction algorithms still require a certain amount of information to produce an accurate result, otherwise there can be numerous possible solutions, and among them just one is considered to be correct. This makes finding the proper angles essentially important in the case of discrete tomography, since we can get entirely different reconstructions from projection sets with even the same number of projections.

There are also theoretical results, giving upper bounds to the number of required projections in case of reconstructing convex objects [6]. Also, one of our longterm goals is to discover a more general description on how determined a binary reconstruction can be by a given set of projections.

The main aim of our current study is to determine, whether the result of a reconstruction can be improved by finding the correct directions to take projections with. If the result can be improved in such a way, one can develop more accurate reconstruction techniques, capable of providing better reconstructions exclusively by finding the appropriate projection angles.

In this paper, we summarize and extend the previous results connected to the angle selection dependency in binary tomography. We conduct experimental software tests on phantom images by reconstructing them from different equiangular and non-equiangular sets of their projections and compare the resulting reconstructions. We do experiments by applying three different binary reconstruction algorithms, and examine the effects of modifications of data, i.e., when the projections are corrupted by noise, or the object to be reconstructed is slightly altered (for example it has fractures or unwanted holes in it). The novelty of our studies lies in examining the case, when we allow the usage of non-equiangular projection sets and in the same time assume presence of distortions of the data. Furthermore, we will give a brief description of the nature of the problem, and describe some of its possible consequences.

Although, we do mention some angle selection strategies in this paper, we must highlight that our goal is not to propose a new reconstruction algorithm, but only 
to evaluate the direction-dependency of three currently existing ones. We must also note that - although our results contain an explicit evaluation of the performance of the three selected reconstruction algorithms - we do not intend to compare them and decide which one is the best.

The paper is structured as follows. In Section 2 we introduce a formulation of the transmission tomography problem, and in Section 3 we present algorithms for solving it in the binary case. In Section 4 we describe the test frameset used for our experiments. In Section 5 we give some of our results, and provide an explanation of them. Finally, in Section 6 we conclude our work, and suggest some possible extensions of it.

\section{Transmission Tomography}

In this chapter we will provide a model of the continuous two-dimensional tomographic reconstruction problem, that will serve as the basis of describing the formulation of the reconstruction problem in the discrete environment.

In a common representation of two-dimensional transmission tomography there is an unknown $f: \mathbb{R}^{2} \rightarrow \mathbb{R}$ function we want to reconstruct (usually, because it represents the two-dimensional cross-section of a real-world object). The only data we can measure about this unknown function is a set of its line integrals given by the Radon-transform as

$$
[R f](\alpha, t)=\int_{-\infty}^{\infty} f(t \cos (\alpha)-q \sin (\alpha), t \sin (\alpha)+q \cos (\alpha)) d q
$$

where the $\alpha$ and $t$ value, respectively, describes the direction and the position of a line in the two-dimensional space, with its points parameterized by $q$. In transmission tomography the task is to reconstruct an $f^{\prime}$ function that has the same projections as the original $f(u, v)$ function, in a set of predefined directions. Theoretically, this problem can be solved by exact mathematical methods when all possible $[R f](\alpha, p)$ values are available $[7,12]$.

Unfortunately, in a practical application we usually can only deal with a finite number of values therefore we have to discretize the model applied for both the projection data and the function to be reconstructed. In the followings, we will assume that the function to be reconstructed has constant values on each unit square shaped region determined by the two-dimensional integer lattice, that is

$$
f(u+a, v+b)=f(u+c, v+d) ; \quad u, v \in \mathbb{Z} ; \quad a, b, c, d \in[0,1)
$$

We will further assume that the function $f$ has a bounded support, therefore without the loss of generality we can say that in (2) $u, v \in\left\{\left[\frac{-n}{2}, \frac{n}{2}\right) \cap \mathbb{Z}\right\}$ with a constant $n$ value. This restriction does not affect the applicability of the model, since in a real-world application we do not have infinitely large objects to deal with. This way, the task can be regarded as the reconstruction of an $n \times n$ pixel-sized image, from its projections. 

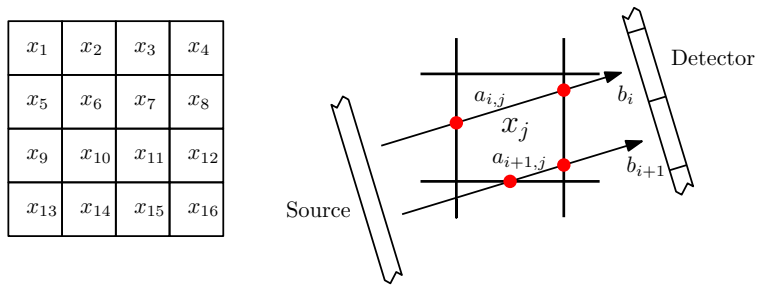

Figure 1: Representation of the ordering of the pixels and the parallel-beam geometry used.

In our experiments a projection was defined as a set of $[R f](\alpha, t)$ values with the same $\alpha$ angles and

$$
t \in\{k+0.5 \quad|k \in \mathbb{N},| k+0.5 \mid \leq n / \sqrt{2}\}
$$

values, assuming that the origin of the coordinate system was placed into the center of the image to be reconstructed. In the defined projection geometry, a projection is composed of integrals taken along a set of equidistantly placed parallel lines. The distance between the neighboring projection lines was set to be 1 unit in the coordinate system and we used as many projection lines as needed to cover the whole image. The rotation center used for controlling the relation of the image and the projections was placed half-way between two projection lines, and in the center of the image to be reconstructed.

Using the previous restrictions the reconstruction problem can be represented by a system of equations

$$
\mathbf{A x}=\mathbf{b} ; \quad \mathbf{A}=\left(a_{i, j}\right)_{m \times n^{2}} \in \mathbb{R}^{m \times n^{2}}, \mathbf{x} \in \mathbb{R}^{n^{2}}, \mathbf{b} \in \mathbb{R}^{m},
$$

where $\mathbf{x}$ denotes the ordered sequence of the pixels of the unknown image to be reconstructed, $\mathbf{b}$ is the sequence of the measured projection values and $\mathbf{A}$ describes the connection between $\mathbf{x}$ and $\mathbf{b}$, where all $a_{i, j}$ elements give the length of the line segment of the $i$-th projection line through the $j$-th pixel. An illustration can be seen in Figure 1. Ideally, by solving equation system (4) we can get the pixel values of an image that has exactly the same projections as the measured ones.

Although, there are several general methods for solving equation systems, a direct method for finding the solutions of (4) is usually not the best approach. The resulting equation system can be too large for exact equation system solvers and might also be underdetermined, possibly yielding an infinite number of solutions. On the other hand, we cannot even guarantee that there is a solution, since - due to measurement errors and noise - the equation system can be inconsistent as well.

The different types of the algebraic reconstruction techniques $[2,7,12]$ try to overcome this problem by applying iterative algorithms to approximate a solution, from a suitably chosen initial suggestion. 
Another group of reconstruction methods reformulate the task as an energy minimization problem with a given

$$
\mathcal{C}(\mathbf{x})=\|\mathbf{A} \mathbf{x}-\mathbf{b}\|_{2}^{2}+\lambda \cdot g(\mathbf{x})
$$

energy function. In the above formulation $\mathbf{A}, \mathbf{b}$, and $\mathbf{x}$ are the same as defined in (4) and $g(\mathbf{x})$ is a function representing additional information about the image to be reconstructed with a given $\lambda$ weight. In the ideal case, we can find a vector $\mathbf{x}^{*}$, where (5) takes its minimal value, with an appropriate general optimizer $[1,14]$. This $\mathrm{x}^{*}$ vector will represent the desired reconstruction. The main advantage of this approach is that it can easily incorporate some a priori information into the model via a suitable $g(\mathbf{x})$ function.

\section{Binary Reconstruction Algorithms}

We examined the projection selection dependency in binary tomography, i.e., in the case when the results can contain only binary values (and $\mathbf{x} \in\{0,1\}^{n^{2}}$ in (4)). In our experiments we applied three suitable binary tomographic reconstruction algorithms from the corresponding literature. All three algorithms are deterministic, therefore their results are unique for each input and can easily be evaluated. The brief introduction of the algorithms can be given as follows.

\subsection{Thresholded Simultaneous Iterative Reconstruction Technique (TSIRT)}

The first algorithm was basically a continuous reconstruction followed by a thresholding. The continuous reconstruction was produced by the Simultaneous Iterative Reconstruction Technique (SIRT) [7, 12], which is an iterative algorithm for finding an approximate solution of (4). After obtaining the continuous result we applied a thresholding of the pixel values with a 0.5 threshold.

\subsection{Discrete Algebraic Reconstruction Technique (DART)}

The second algorithm was the Discrete Algebraic Reconstruction Technique [2], which is a combination of an algebraic based reconstruction method and a iterated thresholding. The DART starts by producing a continuous reconstruction using a suitable algorithm, and applies a thresholding of the result. Later, the values of the boundary pixels are fine-tuned by an iterative process.

In our experiments, we applied 10 iterations of the SIRT algorithm to obtain the continuous reconstructions, and used a threshold value of 0.5.

\subsection{Energy Minimization Tomography with DC Program- ming}

The third algorithm - that was first introduced in [16] - is based on DC programming (a numerical method for minimizing the difference of convex functions), and 
performs the reconstruction by minimizing an energy function given as

$$
\mathcal{J}_{\lambda}(\mathbf{x}):=\|\mathbf{A} \mathbf{x}-\mathbf{b}\|_{2}^{2}+\frac{\gamma}{2} \sum_{j=1}^{n^{2}} \sum_{l \in N_{4}(j)}\left(\mathbf{x}_{j}-\mathbf{x}_{l}\right)^{2}-\lambda \frac{1}{2}\langle\mathbf{x}, \mathbf{x}-\mathbf{e}\rangle, \quad \mathbf{x} \in[0,1]^{n^{2}} .
$$

Here, $\gamma$ is a fixed constant to control the weight of the smoothness term on the result, $N_{4}(j)$ is the set of pixels 4 -connected to the $j$-th pixel, and e denotes the vector with all $n^{2}$ coordinates equal to 1 . This algorithm starts with approximating an optimal continuous result by minimizing the energy function with a $\lambda=0$ value. After, an iterated process forces binary results, by proceeding with the minimization while periodically increasing $\lambda$ with a $\lambda_{\Delta}$ value.

In the sequel, we will simply call this algorithm "DC". In the reconstructions the parameter settings of the DC algorithm were determined as in [17].

\section{Test Frameset}

We conducted experimental tests on a set of software phantoms. We reconstructed them from different sets of their projections and we evaluated the results from two different viewpoints. First, we compared the reconstructions of the same phantoms, under the same conditions but from different sets of their projections. With the result of these experiments, we could determine how dependent the reconstruction of a specific object - performed by a specific algorithm - can be on the choice of projection angles. Secondly, we compared the reconstruction of the same objects, performed by using the same projection directions, but under different conditions (original and altered versions of the objects, addition of different levels of noise, or in case when the reconstruction is performed by different algorithms), in order to see if there are regularities of the projection selection dependency of an object which can be possibly used in practical applications.

Our test database consisted of 10 phantom images, all having the same size of $128 \times 128$ pixels. The database could be divided into two parts, 5 basic phantoms with different properties (those can be seen in Figure 2) and a slightly altered version of each basic phantom (the ones shown in Figure 3), which simulate small distortions of the object of study (i.e., fractures or bubbles).

The elements of the test database were chosen based on previous studies $[2$, 14, 16, 17, 18, 19]. Phantoms like the ones of Figure 2a and Figure 2b, containing circles in a ring, are commonly used test images in discrete tomography, since they are easy to generate but relatively hard to reconstruct. The ones in Figure $2 \mathrm{c}$ and Figure $2 \mathrm{~d}$ are phantoms similar to complex real world objects. Finally, Figure 2e shows a highly direction dependent image, that is useful for illustrating our results.

For simulating measurement errors during the projection acquisition phase of a real-world application, some of the experiments were performed using projection data corrupted by noise. In a real application the gathered projection data can be degraded by different artifacts - e.g.: beam hardening, photon scattering, imperfections of the detectors, background noise, etc. - most of which can be handled 


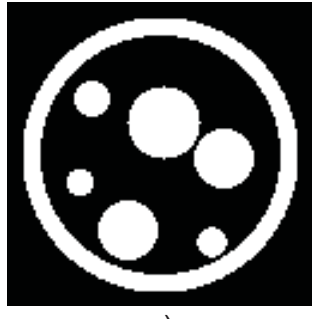

a)

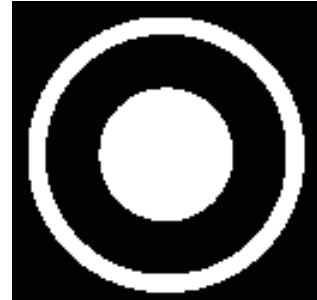

b)

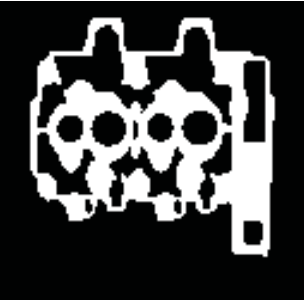

c)

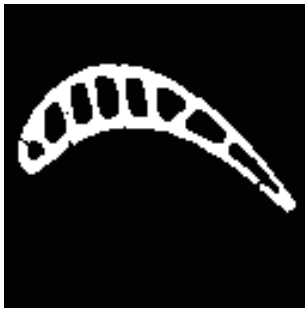

d)

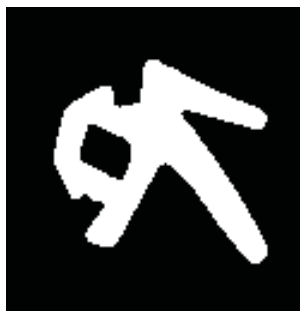

e)

Figure 2: Basic images in the test database.

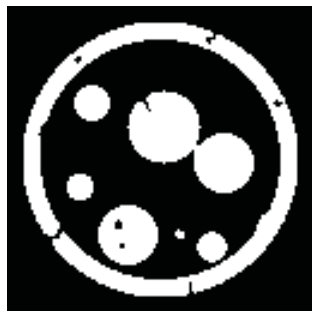

a)

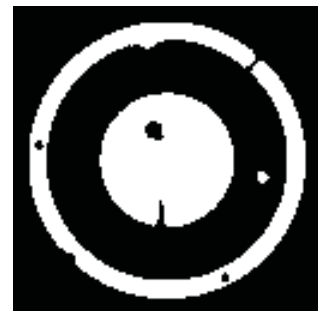

b)

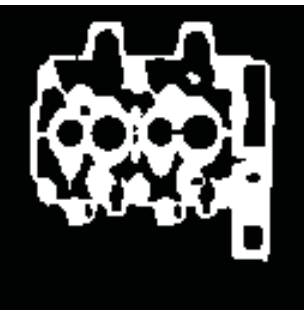

c)

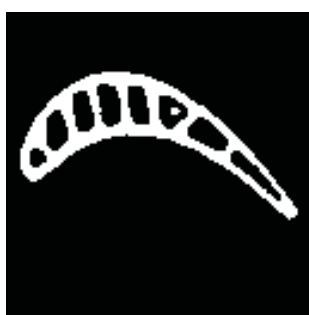

d)

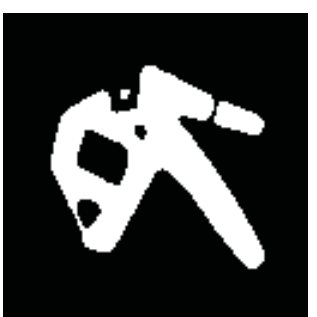

e)

Figure 3: Altered images in the test database. 
by preprocessing steps $[3,5,10,13]$. Unfortunately, it was impossible to simulate all these effects in our experiments. Therefore, we decided to apply an additive Gaussian noise, which is a common technique for modeling noise is transmission tomography $[4,5,13,20]$.

In each case the mean value of the noise was set to $\mu=0$, and the standard deviation was chosen from the set $\sigma \in\{0.5 ; 1.5 ; 5.0\}$. Taken, that the mean of the projection values was approximately 40 , we can say that the amount of noise in the projection was respectively $0 \%, 1.25 \%, 3.75 \%$ and $12.5 \%$, compared to the amount of useful data. Together with the noiseless case, this gave four different versions of the projection data. We generated and stored the noise in advance, before the reconstructions, in order to ensure the same conditions in all experiments.

The reconstruction algorithms were implemented with GPU acceleration using the NVIDIA CUDA programming toolkit [21]. For the computation we used a 2.66 GHz Core 2 Quad CPU, and an NVIDIA GeForce GTS250 GPU. With this highly parallel implementation the time required to perform all 1145224 reconstruction tasks was about 500 hours.

For the evaluation of the results we used a numerical error measurement called Relative Mean Error $(R M E)$ that was defined in [11]. The $R M E$ value of a reconstruction is computed as

$$
\operatorname{RME}\left(\mathbf{x}^{*}, \mathbf{y}\right)=\frac{\sum_{i=1}^{n}\left|x_{i}^{*}-y_{i}\right|}{\sum_{i=1}^{n} x_{i}^{*}} .
$$

Here $\mathbf{x}^{*}$ denotes the vector of pixel values of an expected reconstruction (in our case the pixel values of the phantom processed) and $\mathbf{y}$ is the reconstruction provided by one of the reconstruction algorithms described in Section 3, performed under a specific set of conditions (we will refer to the phantom and its reconstruction given by $\mathbf{x}^{*}$ and $\mathbf{y}$, in the text). Informally, the $R M E$ value gives the ratio of missed pixels in a binary reconstruction, normalized by the number of object pixels on the expected image. The main advantage of the $R M E$ measurement is that it gives the amount of error compared to the size of the object of study, and not the image itself (thus the error measurement is not affected by the zero padding of the image to be reconstructed). As a consequence the $R M E$ measurement can take values higher than 1 , but fortunately this does not affect our evaluations, since we are interested in comparing the reconstructions to each other (and not determining their quality).

We generated the projection sets used in our experiments with two angle set selection techniques described in [18]. In the followings we shortly describe them.

\subsection{Equiangular Angle Sets}

The first type of angle sets were generated equiangularly along the half circle. Such projection angle sets are uniquely determined by their number of $p$ projections and a starting angle $\alpha$ as

$$
S(\alpha, p)=\left\{\alpha+i \frac{180^{\circ}}{p} \mid i=0, \ldots, p-1\right\} .
$$




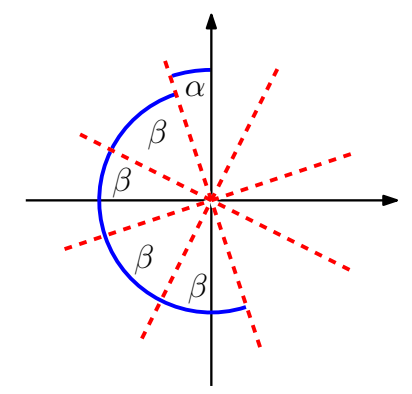

Figure 4: Example of the equiangular projection angle sets (angle set $S(\alpha, 4)$ ).

An example of the equiangular angle sets is given in Figure 4. With our notation $0^{\circ}$ stands for vertical projection beams, aimed from the bottom to the top of the image.

With each image, reconstruction algorithm and specified noise, we performed reconstructions from projection sets $S(\alpha, p)$, with $p \in\{2, \ldots 18\}$ projection numbers and all integer $\alpha$ starting angles ranging between $0^{\circ}$ to $\left\lfloor\frac{180^{\circ}}{p}\right\rfloor$.

\subsection{Angle Set Selection with Greedy Angle Testing}

We also conducted experiments on several non-equiangular projection sets. Unfortunately, the extremely high number of such sets (even assuming only integer angles between $0^{\circ}$ and $179^{\circ}$ with 2 projections would produce $\left(\begin{array}{c}180 \\ 2\end{array}\right)=16110$ possible angle sets) made it impossible to perform such a thorough testing that we did in the equiangular case. Therefore, we had to choose a smaller subset of all the possibilities.

In accordance to practical applications we restricted our studies to projection sets producing highly accurate results. We must note that the performance of projection sets strongly depends on the image to be reconstructed, and other conditions - like the applied reconstruction algorithm or noise - can also have influence on the information content of the projections. Therefore, we generated non-equiangular angle sets for each image, algorithm and noise level, trying to find highly accurate projections sets.

For the purpose of generating the desired angle sets we used the greedy angle selection algorithm of [18], that produces an $L=\left\langle\alpha_{1}, \alpha_{2}, \ldots, \alpha_{p}\right\rangle$ ordered list of angles as follows.

Greedy: Greedy angle selection algorithm.

Input: $\mathbf{x}^{*}$ vector of image pixel values, $p \geq 2$ maximal number of angles, and $1 \leq \alpha_{1} \leq 179$ predetermined integer angle.

Output: $\bar{L}=\left\langle\alpha_{1}, \alpha_{2}, \ldots, \alpha_{l}\right\rangle$ angle list so that $l \leq p$.

Step 1 Set $L_{1}=\left\langle\alpha_{1}\right\rangle, i=1$; 


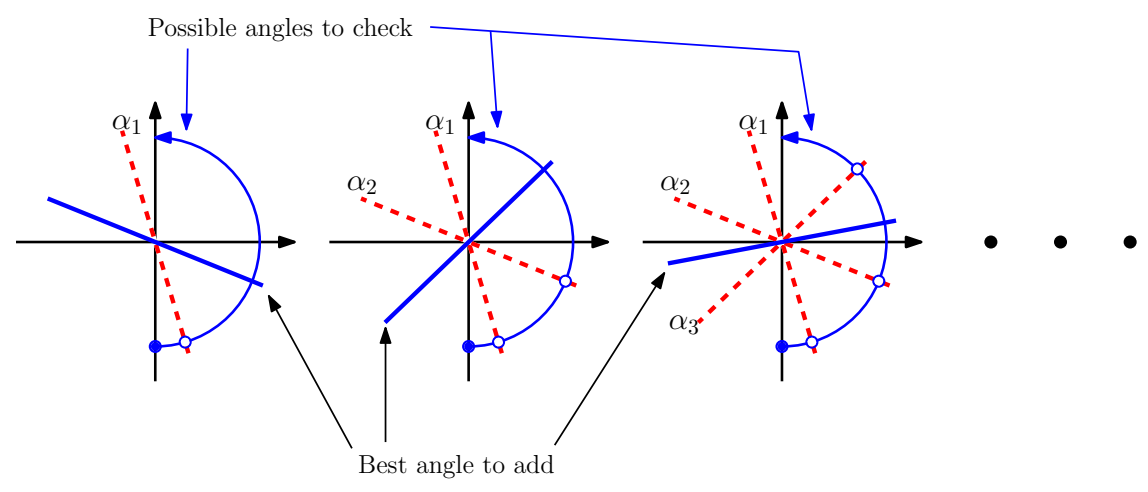

Figure 5: Steps of the Greedy algorithm.

Step 2 Let $i \leftarrow i+1$;

Step 3 Let $0^{\circ} \leq \alpha^{*} \leq 179^{\circ}$ be an integer angle for which $R M E\left(\mathbf{x}^{*}, \mathbf{x}_{\left\langle L_{i-1}, \alpha^{*}\right\rangle}\right)$ is minimal;

Step 4 Let the next list of angles be $L_{i}=\left\langle L_{i-1}, \alpha^{*}\right\rangle$;

Step 5 If $i=p$ or $R M E\left(\mathbf{x}^{*}, \mathbf{x}_{L_{i}}\right)=0$ return with $L_{i}$ otherwise go to Step 2

Here, $\mathbf{x}_{L}$ stands for the reconstruction of the $\mathbf{x}^{*}$ image from the projection set specified by the angles of $L$.

The Greedy algorithm is an iterative process that takes an image, and in each iteration it tries to determine the best projection to be added to the current ones. The result of this algorithm is a list of angles ordered by decreasing significance. A demonstration of the algorithm's proceeding is plotted in Figure 5.

In each iteration, when there are $i$ projections already chosen, the algorithm has to test $180-i$ possible projection sets to find the best one to proceed with. This determines a total number of $\sum_{i=1}^{p-1}(180-i)$ projection angle sets, assuming that the maximal number of projections is $p$. We used these angle sets for building up our test frameset. This provided reconstructions from a significant number of non-equiangular projection sets, that gave a proper base for comparing the results of the different reconstruction algorithms.

Although, in [18] other methods for non-equiangular angle set selection are also described - which could have been used for generating the desired angle sets in our studies - we decided to use the Greedy algorithm, due to its deterministic nature and relatively fast performance.

The parameters of the Greedy algorithm were set as described in [18]: we allowed to choose integer angles between $0^{\circ}$ and $179^{\circ}$, and used the SIRT algorithm for producing the first $\alpha_{1}$ projection angle. Furthermore, we set the maximal number of projections to 18 . 


\section{$5 \quad$ Experimental Results}

After performing the tests we started comparing the $R M E$ values of the reconstructions. As it was mentioned in Section 4 we evaluated the data from two viewpoints. First, we compared the reconstructions of the same objects, performed under the same conditions (i.e., using the same reconstruction algorithms and same noise), but from different projection sets to see how much improvement can be reached in the reconstruction, solely by finding better projections. Secondly, we compared the reconstructions of the same objects, from the same projections, but under different conditions to find out whether or not the direction dependency of the objects remain consistent under different conditions - i.e., to see if there are projection angle sets which lead to better (or worse) reconstruction results under all circumstances.

Although all the results proved our observations, due to the extremely high number of performed reconstructions we cannot present all of them in detail. Therefore, in this section we will discuss our general observations, and show only samples of the result dataset which demonstrate them the best.

\subsection{Reconstruction from Equiangular Projection Sets}

In the case of the equiangular angle sets the task was relatively easy. We could plot the curves of $R M E$ values belonging to the reconstructions performed by one of the three reconstruction algorithms, from a specific number of projections, and using a given type of noise, according to the starting angle (as it was done in [19]). Then, we could easily determine if the curves have significant differences between their minimal and maximal values (i.e., if the accuracy of the reconstructions depend on the choice of the projection angles), and if the curves on the diagrams are similar or not (i.e., if there is correspondence between the reconstructions of the same object from the same projections, but under different conditions). As an example, the $R M E$ values belonging to the reconstructions of the phantoms in Figure 2e and Figure 3e (the basic and altered versions of the same object) can be seen in Figure 6. The plotted graphs show statistics of all the reconstructions performed from equiangular projection sets containing 4 projections, grouped by the different types of applied random noise.

Apparently, all the graphs have significant gaps between their minimum and maximum points, showing that the binary reconstruction of an object can indeed be improved only by taking the proper projections (Figures 7 and 8 give examples of the reconstructed images preformed with different projection sets). We can also notice, that the curves of the graphs are relatively smooth, suggesting that projections with angles close to each other have similar information content. This also indicates that small changes of the projection angles may only have negligible effects on the result of the reconstruction. In addition, all the curves show some degree of similarity, i.e., the minimal and maximal values correspond to similar projection angle sets and the transitions between the extrema are also alike.

Such examination of equiangular projections has already been done in $[17,18]$, but we decided to reproduce these results to highlight the regularities and give a 

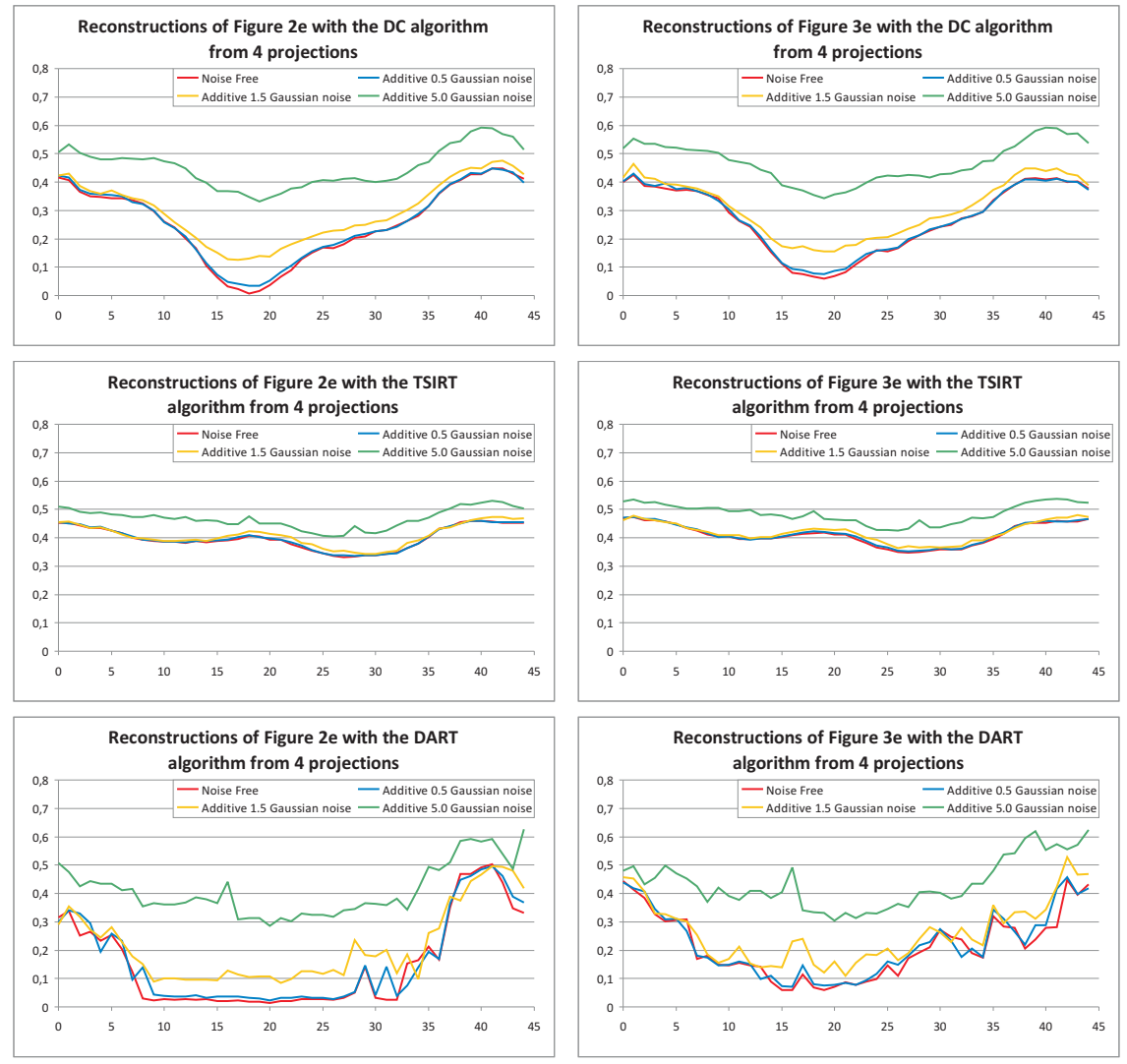

Figure 6: $R M E$ values of the reconstructions of the phantoms in Figure 2e and Figure $3 \mathrm{e}$ from 4 projections according to the starting angle. The three diagrams on the left hand side are the reconstructions of Figure 2e, and those on the right hand side are the reconstructions of Figure 3e. Each row shows the results of one applied reconstruction algorithm (from the top to the bottom: DC, TSIRT and DART algorithms, respectively). Each diagram shows four curves each belonging to the reconstructions affected by the four different types of noise. On the diagrams: horizontal axis stands for the starting angle, and vertical axis for the $R M E$ value. 
brief explanation of the projection selection dependency of objects.

Let us consider the problem of angle selection dependency in general, i.e., without the assumption of examining any specific object, reconstruction algorithm, or angle selection strategy. As it was described in Section 2, the goal of transmission tomography is to reconstruct a function $f^{\prime}$, that has the prescribed projections in a set of directions. We also mentioned that the limited amount of projection values may not contain enough information for a proper reconstruction. This lack of data makes it possible to have several different $f^{\prime}$ functions having the same projections, i.e., different reconstructions. Nevertheless, we are usually interested in only one specific result (the one identical to the original object), and any other solution is considered to be incorrect.

In our discretized grid based representation, the lack of information means that there can be several different binary images having the expected projections, some of which can be entirely different from the expected reconstruction. In this case the most we can expect from a reconstruction algorithm is to find one of these possible solutions. If the set of possible reconstructions is large, the probability of finding an accurate solution can be small. Naturally, if we want to increase the probability of finding an acceptable reconstruction, we have to reduce the set of possibilities, by gaining additional information for the reconstruction. This can be done by either adding some extra prior knowledge to the model (e.g. by assuming that the shape of the object of study fulfils some special property [9]), acquiring additional projections, or taking better projections with higher information content.

Since we have been experimenting on deterministic algorithms, the choice of the reconstruction method can also be regarded as a prior knowledge, i.e., to decide which strategy should be used for choosing a reconstruction out of the possible ones.

Regarding the previous discussion, the differences in the curves of Figure 6 can easily be explained. First, let us consider the effects of the additive noise. In this case changing the projection data also affects the feasible solutions the algorithms can choose from, and all the possible solutions will have some degree of error. Since we applied the same noise each time, the distortion of data is similar with every reconstruction and the effect in Figure 6 is an approximately constant upwards shifting in the $R M E$ value curves. Naturally, higher noise levels result in bigger upwards shifting of the curves.

The effect is different when we compare the RME curves on Figure 6, belonging to the basic and altered version of the phantoms. We know, that some objects can be reconstructed easier if their projections are taken from a specific set of directions. It is also clear, that different objects can have different optimal angle sets. Therefore, we can only find the optimal set of directions for a specific object. If we alter the structure of the the object, the information content of its projections taken from specific directions can change, thus some of them can be more (or less) useful for the reconstruction. This means that the modified object can have different optimal angle sets. Our results show that this change of the optimal directions correspond to the level of modification of the object. Small modifications do not notably affect the result of reconstruction, but by increasing the distortion we reach 


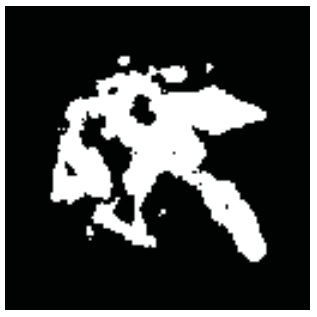

a)

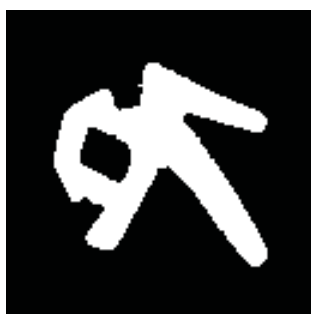

d)

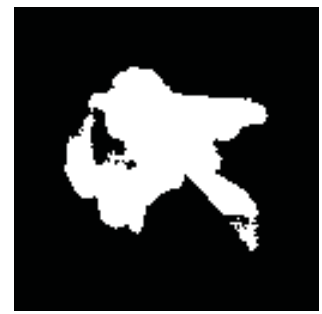

b)

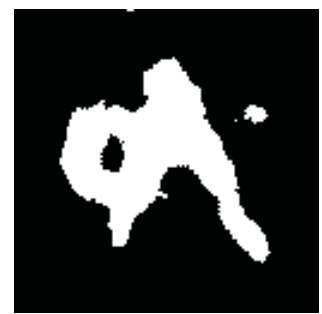

e)

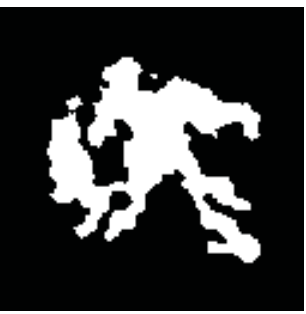

c)

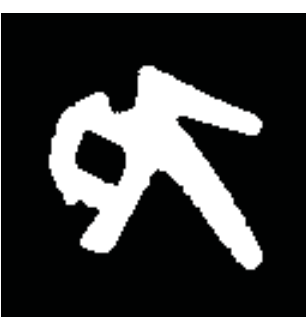

f)

Figure 7: Reconstructions of the phantom in Figure 2e performed by the three reconstruction algorithms, from different projection sets containing 4 projections. Images a, b, c correspond to the worst, and d, e, f to the best reconstructions gained by using equiangular projection sets. The three columns contain results belonging to the different reconstruction algorithms (DC: a, d; DART: b, e; TSIRT: c, f ).

a certain point, where we are dealing with an entirely different object, with different properties.

The differences in the curves of Figure 6 are the most remarkable in the case of comparing the results belonging to the different reconstruction algorithms. Since we used the same information in all the reconstructions (the projection data and the fact that we are looking for binary solutions), the choice of the reconstruction algorithm should only influence which one of the feasible results is found. This previous assumption is, however, not true, since the algorithms we used are not guaranteed to give optimal solutions. Still, having more informative projection sets can make it more likely to get better results from the same projection sets regardless of the applied reconstruction algorithm.

We should also take a look at what this projection angle dependency means regarding the reconstructed images themselves. In Figure 7 we gave the best and worst reconstructions of the phantom in Figure 2e reconstructed by the three examined algorithms from equiangular projection sets containing 4 projections. Here, we can see significant differences, especially on the reconstructions performed by the DC and the DART algorithms, but even the results of the TSIRT algorithm can notably be improved by finding the proper projections. 


\subsection{Reconstruction from Non-Equiangular Projection Sets}

It was also shown [18] that - in the case of noiseless projections - the dissimilarity between the result of the reconstructions using different projection sets can be even more significant if we allow the acquisition through non-equiangular projection sets. Here, we extended the previous work by examining the case when the projection data is affected by random noise. A brief example this can be seen in Figure 8, showing the reconstructions of the phantom of Figure $3 \mathrm{e}$, from different projection sets. Our results indicate that the previous observations still hold, even in the case of distorted data, and loosing the assumption of equiangularity can bring further improvement to the reconstruction.

Comparing the results numerically is a bit harder when using non-equiangular angle sets, than with the equiangular ones. In this case we could only compare a set of reconstruction pairs by plotting both $R M E$ values of the corresponding reconstructions. We used this technique for a pairwise comparison of the different reconstruction algorithms. Examples for the resulting diagrams can be seen in Figure 9 .

We would expect Figure 9 to show some degree of correspondence between the reconstructions of the different algorithms. If it is so, then the projection sets producing better results for one algorithm should also produce high quality reconstructions for other ones, and the points in the diagrams should be placed along - or at least close to - a diagonal straight line. As we can see, the points of Figure 9 do satisfy our expectations so we can deduce that there is a strong correspondence between the results of the different reconstruction algorithms.

We used the same technique for comparing the reconstructions of the basic and altered versions of the images from projection sets using the same angles, in the case when we allow non-equiangular angles and the projection data can be corrupted by noise. An example of such results is given in Figure 10 by giving the $R M E$ value pairs of the reconstructions of Figure 2e and Figure 3e, performed with the DC reconstruction algorithm. Again, we can see that the points highlighted in Figure 10 are allocated close to a diagonal straight line indicating that small distortions of the object of study do not have a significant influence on the reconstruction. Moreover, we also can deduce that such distortions do not affect the information content of projections taken from specific angles, i.e., if a projection of an object holds high information content, then another projection taken from the same direction, but from a slightly different object would also provide similarly useful information for the reconstruction.

The results indicate that there is a strong correspondence between the binary reconstructions of an object performed with different reconstruction algorithms, even in the case when the projections are chosen non-equiangularly, and the projection data is affected by different types of random noise. Furthermore, we can say that the projection angle dependency of objects remains consistent and somewhat predictable under different conditions, and therefore it is possible to use the direction-dependency of objects in practical applications for improving the results of reconstruction (like it was proposed in $[17,19]$ ). 


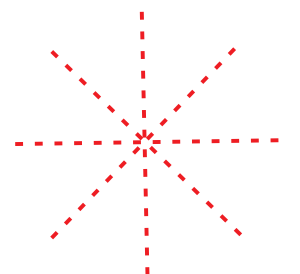

a)

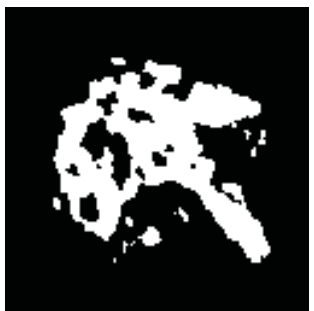

d)

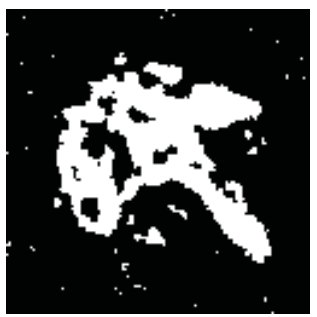

g)

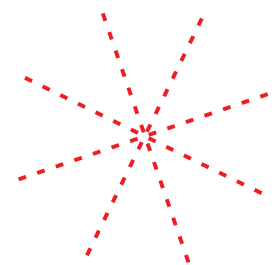

b)

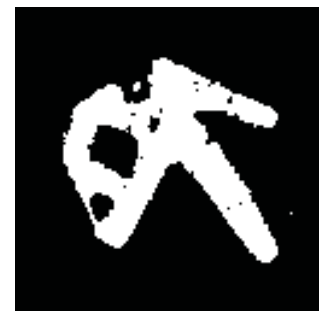

e)

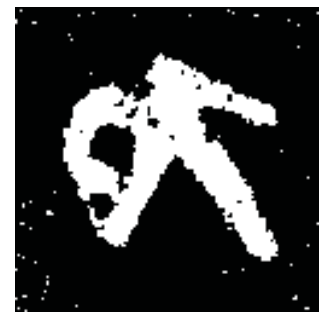

h)

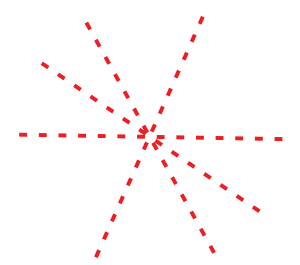

c)

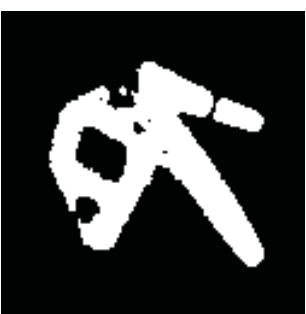

f)

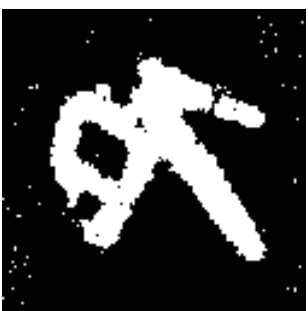

i)

Figure 8: Comparing of the reconstructions of the phantom in Figure 3e, reconstructed by the DC algorithm, from different projection sets, with different levels of noise. Each column contains the angles of the projection sets and the corresponding reconstructions. Top row indicates the angles in the projection sets, second row gives the reconstruction in the noiseless case, bottom row contains the reconstructions from projection sets affected by random noise with 1.5 deviance. 

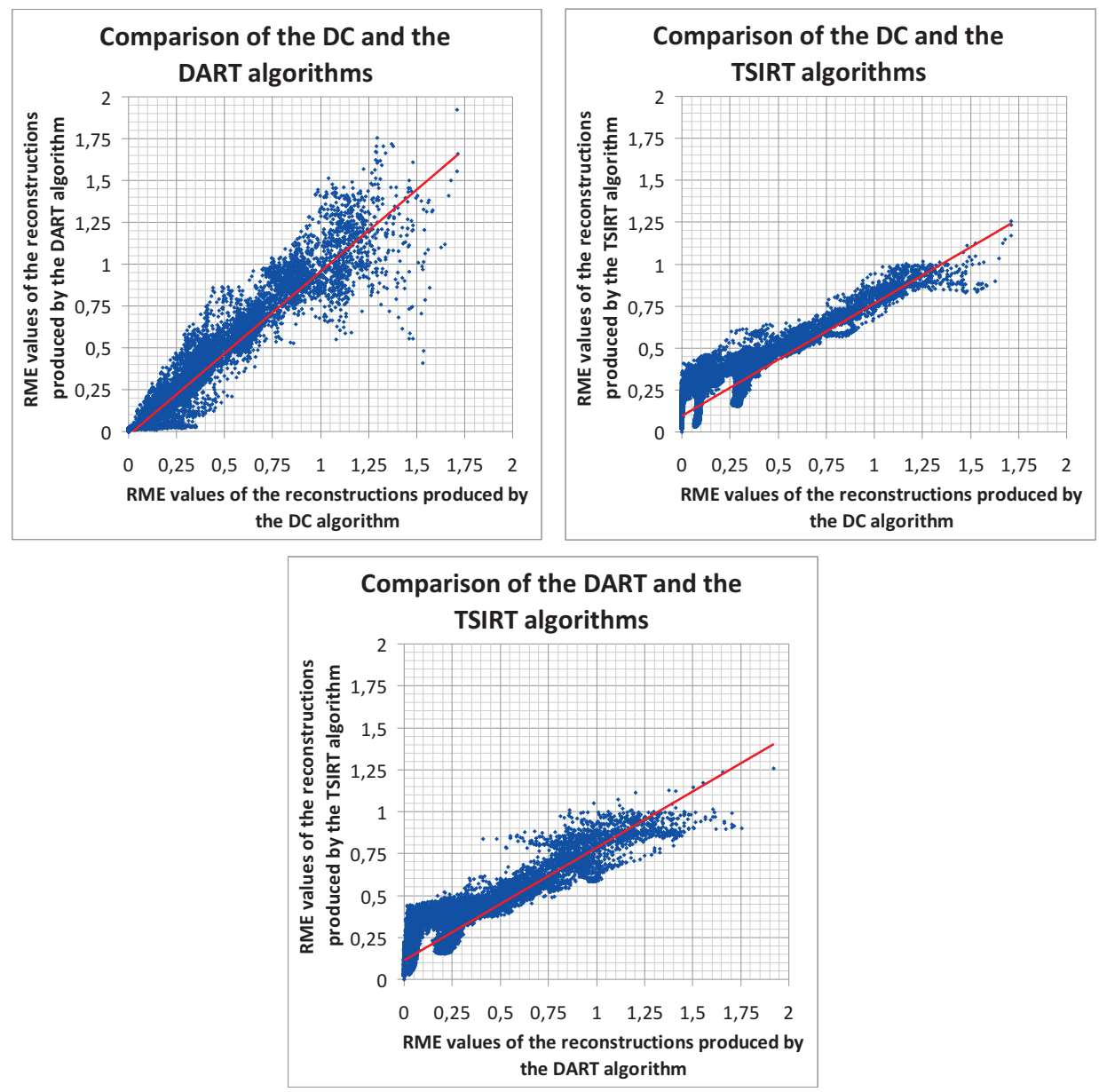

Figure 9: Cross comparison of the algorithms of Section 3 for the reconstructions of the phantom in Figure 2e. Each diagram contains points representing $R M E$ value pairs corresponding to reconstruction performed by two algorithms. The graphs contain results with projection numbers from 2 to 18, and all the additive random noise described in Section 4. The red lines are regression lines fitted to the points for better visibility. 


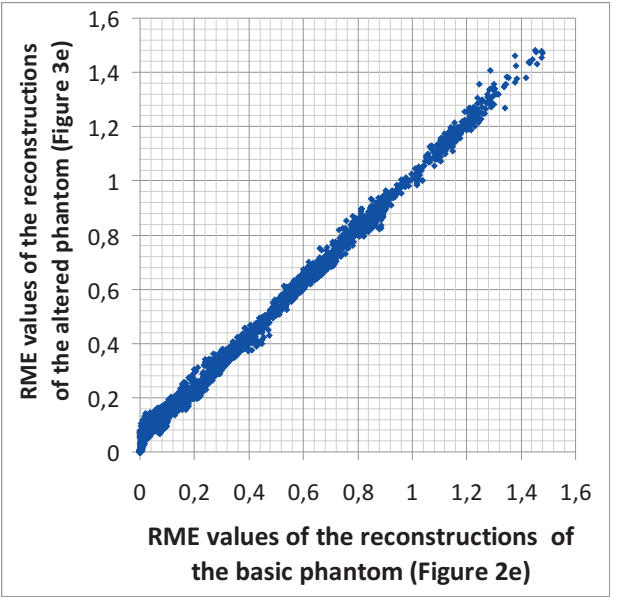

Figure 10: Reconstructions of Figure 2e and Figure 3e performed with the DC reconstruction algorithm from projection sets acquired with different angles. Each point of the diagram is positioned according to the $R M E$ values of the reconstructions of the two images from the projection sets having the same angles. Different points of the diagram give reconstructions corresponding to different angle sets. The diagram also contain reconstructions performed with noise-corrupted projections. 
Although, we have only given a sample of the performed reconstructions, we must note that our observations seemed to hold in all our many test cases.

\section{Conclusion}

In this paper we studied the projection angle selection dependency of reconstructions in the field of binary tomography. We have summarized and extended previous results by showing, that there is a strict correspondence between the reconstructions performed by different binary tomography reconstruction algorithms even in the case when the projection angles are selected from arbitrary directions, the objects of study are distorted and the projection data is affected by random noise.

Our results indicate that this angle selection dependency is caused by the different information content of the different projections, which is the intrinsic property of the images themselves. In the future we intend to discover the deep connections between our experimental results and the theory of discrete tomography, and also to extend our investigations to the case of three dimensional tomography, i.e, when the objects to be reconstructed are represented in three dimensions, and we can take projections from any directions on the sphere.

\section{Acknowledgments}

We would like to thank Joost Batenburg for providing test images (Figures 2c-d and Figures 3c-d) for the studies. The phantom of Figure 2e and Figure 3e were originated from the image database of the IAPR Technical Committee on Discrete Geometry (TC18). We are also grateful to the reviewers of the manuscript for their valuable comments.

\section{References}

[1] P. Balázs, M. Gara, An evolutionary approach for object-based image reconstruction using learnt priors, Lecture Notes in Computer Science 5575 (2009) $520-529$.

[2] K.J. Batenburg, J. Sijbers, DART: a fast heuristic algebraic reconstruction algorithm for discrete tomography, IEEE Conference on Image Processing IV (2007) 133-136.

[3] J. Baumann, Z. Kiss, S. Krimmel, A. Kuba, A. Nagy, L. Rodek, B. Schillinger, J. Stephan, Discrete Tomography Methods for Nondestructive Testing, Chapter 14 of [9] (2007) 303-331.

[4] B. Chalmond, F Coldefy, B. Lavayssière, Tomographic reconstruction from non-calibrated noisy projections in non-destructive evaluation, Inverse Problems 15 (1999) 399-411. 
[5] P. Duvauchelle, N. Freud, V. Kaftandjian, D. Babot, A computer code to simulate X-ray imaging techniques, Nuclear Instruments and Methods in Physics Research B 170 (2000) 245-258.

[6] R.J. Gardner, P. Gritzmann, Discrete tomography: determination of finite sets by X-rays, Transactions of the American Mathematical Society, 349(6) (1997) $2271-2295$.

[7] G.T. Herman, Fundamentals of Computerized Tomography: Image Reconstruction from Projections, 2nd Edition, Springer (2009).

[8] G.T. Herman, A. Kuba (Eds.), Discrete Tomography: Foundations, Algorithms and Applications, Birkhäuser, Boston (1999).

[9] G.T. Herman, A. Kuba (Eds.), Advances in Discrete Tomography and Its Applications, Birkhäuser, Boston (2007).

[10] S. Kimmel, J. Baumann, Z. Kiss, A. Kuba, A. Nagy, J. Stephan, Discrete tomography for reconstruction from limited view angles in non-destructive testing, Electronic Notes in Discrete Mathematics 20 (2005) 455-474.

[11] A. Kuba, G.T. Herman, S. Matej, A. Todd-Pokropek: Medical applications of discrete tomography, Discrete Mathematical Problems with Medical Applications, DIMACS Series in Discrete Mathematics and Theoretical Computer Science, AMS, 55 (2000) 195-208.

[12] A. C. Kak, M. Slaney, Principles of Computerized Tomographic Imaging, IEEE Press, New York (1999).

[13] N.D.A. Mascerenhas, C.A.N. Santos, P.E. Cruvinel, Transmission tomography under Poisson noise using the Anscombe transformation and Wiener filtering of the projections, Nuclear Instruments and Methods in Physics Research A 423 (1999) 265-271.

[14] A. Nagy, A. Kuba, Reconstruction of binary matrices from fan-beam projections, Acta Cybernetica, 17(2) (2005) 359-385.

[15] G. Placidi, M. Alecci, A. Sotgiu, Theory of adaptive acquisition method for image reconstruction from projections and application to EPR imaging, Journal of Magnetic Resonance, Series B, (1995) 50-57.

[16] T. Schüle, C. Schnörr, S. Weber, J. Hornegger, Discrete tomography by convexconcave regularization and D.C. programming, Discrete Applied Mathematics 151 (2005) 229-243.

[17] L. Varga, P. Balázs, A. Nagy, Direction-dependency of a binary tomographic reconstruction algorithm, Lecture Notes in Computer Science 6026 (2010) 242-253. 
[18] L. Varga, P. Balázs, A. Nagy, Projection selection algorithms for discrete tomography, Lecture Notes in Computer Science 6474 (2010) 390-401.

[19] L. Varga, P. Balázs, A. Nagy, Direction-dependency of binary tomographic reconstruction algorithms, Submitted to Graphical Models (CompIMAGE 2010 special issue).

[20] S. Weber, A. Nagy, T. Schüle, C. Schnörr, A. Kuba, A benchmark evaluation of large-scale optimization approaches to binary tomography, Lecture Notes in Computer Science 4245 (2006) 146-156.

[21] NVIDIA CUDA Programming Guide, Version 2.0

http://developer.download.nvidia.com/compute/cuda/2_0/docs/ NVIDIA_CUDA_Programming_Guide_2.0.pdf 